BULL. AUSTRAL. MATH. SOC.

VOL. $29(1984), 357-364$.

\title{
AN INTEGRAL CHARACTERIZATION \\ OF EUCLIDEAN SPACE
}

\author{
J.M. BORWEIN
}

\begin{abstract}
We show that recent integral versions of the classic Jordan-Von Neumann characterization of Euclidean space may be viewed as special cases of a general averaging principle over sets of isometries.
\end{abstract}

1 .

Recently Stanojević and Suchanek [6] showed that a complex normed space $X$ is an inner product space if and only if, given a compact group $G$ with normalized Haar measure $m$ and some non-trivial group character $\gamma$ (that is a continuous homomorphism into the circle group),

$$
\int_{G}\|x+\gamma(g) y\|^{2} d m=\|x\|^{2}+\|y\|^{2}
$$

for all $x$ and $y$ in $X$. Day [3] observed that it suffices for (1) to hold with $X$ replaced by its unit sphere $S(X)$ and with $"="$ replaced by " $\sim$ " where $\sim$ is one of $\leq, \geq$ or $=$. This then gives a broad generalization of the classic Schoenberg-Day characterizations of inner product spaces [2]. In this paper we show that (1) can be viewed as a special instance of an averaging condition involving sets of isometries.

Received 5 December 1983. Research partially supported by an NSERC Grant. Thanks are due to S. Swaminathan and D. Tingley for several stimulating discussions on this subject.

Copyright Clearance Centre, Inc. Serial-fee code: 0004-9727/84 $\$ A 2.00+0.00$. 
2.

Let $X$ be a (real or complex) normed space and let $H$ be a non-empty set of linear isometries. This is to say that $\|T x\|=\|x\|$ for all $T$ in $H$ and all $x$ in $X$. Let $m$ be a Borel probability measure on $H$ (in the induced strong operator topology). We say that $H$ is m-balanced if the barycentre of $H$ with respect to $m$, denoted $m_{H}$, exists and satisfies

$$
m_{H}:=\int_{T \in H} T d m=0
$$

The integral in (2) is interpreted as a weak integral [5] and will exist whenever $H$ is relatively compact and so whenever $H$ is finite dimensional or compact. The next proposition motivates the definition.

PROPOSITION 1. Let $H$ be a non-empty subset of the isometries of an inner product space $X$. Let $m$ be a Borel probability measure on $H$ with respect to which $H$ has a barycentre. Then for $x$ and $y$ in $X$,

$$
\int_{T \in H}\|x+T y\|^{2} d m=\|x\|^{2}+\|y\|^{2}+2 \operatorname{Re}\left\langle m_{H} y, x\right\rangle \text {. }
$$

In particular,

$$
\int_{T \in H}\|x+T y\|^{2} d m \sim 2 \text { for } x, y \text { in } S(X)
$$

if and only if $H$ is m-balanced.

Proof. First observe that $\|x+T y\|^{2}$ is continuous and is bounded by 4. The integral in (3) is thus well defined. Equation (3) is now a consequence of the fact that $X$ is an inner product space, that $H$ contains only isometries, and that $m_{H}:=\int_{T \in H} T d m$. If $m_{H}=0$, (4) follows easily. Conversely, if $m_{H}$ is non-zero one can find a unit vector $y$ with $m_{H} y \neq 0$. If we let $x:= \pm m_{H} y /\left\|m_{H} y\right\|$, (3) shows that (4) is violated.

It is standard and easily observed that $m_{H} \in \overline{\operatorname{conv}} H$. Thus when $H$ is finite dimensional and closed, $H$ is $m$-balanced with respect to some $m$ if and only if $0 \in \operatorname{conv} H$. It follows that certain groups of 
isometries can never be used to obtain expressions like (4). For example, the isometry $T$ of $l_{2}^{n}(R)$ which is given by

$$
T\left(x_{1}, x_{2}, \ldots, x_{n}\right):=\left(x_{2}, x_{3}, \ldots, x_{n}, x_{1}\right)
$$

generates a cyclic group of order $n$ whose convex hull does not contain zero. Recall that a Borel measure on $H$ is strictly positive if its support is $H$. Our main result is :

THEOREM 2. Let $H$ be a subset of the isometries of a finite dimensional normed space $X$. Suppose that $H$ is m-balanced with respect to a strictly positive measure $m$. If

$$
\int_{T \in H}\|x+T y\|^{2} d m \sim 2 \text { for } x, y \text { in } S(X),
$$

then $X$ is a Euclidean space.

Proof. A complex normed space of dimension $n$ may be viewed (real isometrically) as a real normed space of dimension $2 n$. The complex isometries remain real isometries, and since (5) is a real isometric invariant, it suffices to establish the real case of the theorem. We consider the " $\geq$ " case and let $E$ be the unique (Loewner) ellipsoid of maximal volume inside $C:=\{x \mid\|x\|=1\}$. Let $\|\cdot\|_{E}$ denote the associated Euclidean norm. The argument in $[1$, p. 90$]$ shows that $E$ inherits the isometries of $X$; and [2, p. 80] shows that $M:=S(E) \cap S(X)$ spans $X$. Let $x$ and $y$ lie in $M$ and choose $T_{0}$ in $H$. Then $z:=T_{0} x$ also lies in $M$ and (5) shows that

$$
\int_{T \in H}\|z+T y\|^{2} d m \geq 2
$$

Since $H$ is $m$-balanced and lies inside of the isometries of $E$, (3) shows that

$$
\int_{T \in H}\|z+T y\|_{E}^{2} d m=2
$$

Let $f(T):=\|z+T y\|^{2}-\|z+T y\|_{E}^{2}$. Then $f$ is a non-positive continuous function on $H$ such that $\int_{T \in H} f(T) d m \geq 0$. Since $m$ is strictly 
positive we must have $f\left(T_{0}\right)=0$. But this says that

$$
\|x+y\|=\left\|T_{0} x+T_{0} y\right\|=\left\|T_{0} x+T_{0} y\right\|_{E}=\|x+y\|_{E},
$$

because $T_{0}$ is an isometry of $C$ and of $E$. It follows that the set of directions $D$ in which the two norms coincide is midpoint-convex. Being closed and homogeneous, $D$ must actually be a subspace. Since $D$ contains $M, D$ is the entire space and $\|\cdot\|$ coincides with $\|\cdot\|_{E}$. The "s" case follows similarly from a minimality argument.

We can replace the finite dimensionality hypothesis by the condition that, for some fixed $n, H$ leaves $n$-dimensional subspaces of $X$ invariant. This still allows us to show that every $n$-dimensional subspace of $X$ is Euclidean; and so is $X$.

We also observe that the previous argument fails for any skew-norm.

The classical criterion uses $H:=\{I,-I\}$ and the uniform two-point measure. More generally we have:

COROLLARY 3. Let $X$ be a finite dimensional normed space and let $H$ be a closed subgroup of isometries which contains a non-trivial multiplication. Let $m$ be normalized (left) Haar measure on $H$. Then $H$ is m-balanced and (5) characterizes Euclidean space.

Proof. Let $S$ be a multiplication by $\alpha(\alpha \neq 1)$ which lies in $H$. Then $H$ is compact, whence $m_{H}$ exists and

$$
m_{H}=\int_{T \in H} T d m=\int_{T \in H} S T d m=\alpha m_{H} .
$$

Since $\alpha \neq 1, m_{H}=0$ and $H$ is m-balanced. Also, since $H$ is compact and $m$ is translation invariant, $m$ is strictly positive. The result now follows from Theorem 2 . $\quad$ a

A simple way of guaranteeing that a group $H$ is balanced is to require that $H=-H$. Note that the full group of isometries is balanced. Our next corollary recaptures Day's version [3] of Stanojevic and Suchanek's result [6] given in the introduction. Applications can be found in [6]. Observe that only abelian compact groups really appear in the corollary. 
COROLLARY 4. Let $X$ be a complex normed space and let $G$ be a compact group endowed with normalized Haar measure. Let $\gamma$ be a nontrivial group character on $G$. Then

$$
\int_{g \in G}\|x+\gamma(g) y\|^{2} d m \sim 2 \text { for alz } x, y \in S(X)
$$

if and only if $X$ is an inner product space.

Proof. For each $g$ in $G$ multiplication by $\gamma(g)$ is an isometry of $X$. Since $G$ is compact the character $\gamma$ induces a compact subgroup $H$ of isometries of $X$. Since $\gamma$ is non-trivial, $H$ contains a non-trivial multiplication and, as in the previous corollary, is m-balanced.

Proposition 1 now shows (10) to be necessary; and Theorem 2, which applies since $H$ has one dimensional orbits, shows (10) to be sufficient.

If one defines characters with respect to the underlying scalar field, Corollary 4 remains valid - if uninteresting - over the real field. Similarly, we have:

COROLLARY 5. Let $X$ be a normed space, Suppose that wnit length scalars $w_{1}, w_{2}, \ldots, w_{m}$ and strictly positive real numbers $\lambda_{1}, \lambda_{2}, \ldots, \lambda_{m}$ are given such that

$$
\sum_{i=0}^{m} \lambda_{i} w_{i}=0, \sum_{i=1}^{m} \lambda_{i}=1 .
$$

Then $X$ is an inner product space if and only if

$$
\sum_{i=1}^{m} \lambda_{i}\left\|x+w_{i} y\right\|^{2} \sim 2 \text { for } x, y \text { in } s(X) \text {. }
$$

Proof. Let $H$ be the finite set of isometries $T_{i}$ with $T_{i} x:=w_{i} x$. Condition (11) shows that the discrete measure $m$ with mass $\lambda_{i}$ at $T_{i}$ balances $H$, and is strictly positive. The result now follows as in Corollary 4.

The proof is unchanged if $m=\infty$.

A special case of Corollary 5 (and of Corollary 4) is worth singling 
out. If $w \neq 1$ is any $m$ th root of unity then $\sum_{i=1}^{m} \frac{1}{m} w^{i}=0$, and

$$
\frac{1}{m} \sum_{i=1}^{m}\left\|x+w^{i} y\right\|^{2} \sim 2 \text { for } x, y \in S(x)
$$

characterizes inner-product spaces as in [3].

Similar extensions can be made to the integral inequalities given in [4] and extended to group characters in [3]. They do not, however, have the same completeness or simplicity as Theorem 2. Also, it seems worth observing that integration over a Haar measure gives a concise proof of the following classical result.

THEOREM 6 ([1]). Let $c$ be a convex body in finite dimensional normed space $X$. If any two points on the boundary of $C$ are connected by a linear isometry then $C$ is an ellipsoid.

Proof. Let $\|\cdot\|_{E}$ be any Euclidean norm on $X$. Let $H$ be the compact group of isometries on $C$ and let $\|\cdot\|_{F}$ be defined by

$$
\|x\|_{E}^{2}=\int_{T \in H}\|T x\|_{E}^{2} d m
$$

where $m$ is Haar measure on $H$. Then $\|\cdot\|_{F}$ is Euclidean and $\|T x\|_{F}=\|x\|_{F}$ for each $x$ in $X$ and each $T$ in $H$. Since $H$ is transitive, all points on the boundary of $C$ have the same value under $\|\cdot\|_{F}$. Thus $C$ is an ellipsoid.

In the symmetric case the corollary follows easily from our results. Let $H$ be the full group of isometries of $X$ and let $m$ be haar measure on $H$. Define $\Phi$ by

$$
\Phi(x, y):=\int_{T \in H}\|x+T y\|^{2} d m \text { for } x, y \in S(X) .
$$

If $S_{1}$ and $S_{2}$ are isometries in $H$ then

$$
\Phi\left(S_{1} x, S_{2} y\right)=\Phi\left(x, S_{2} y\right)=\Phi(x, y)
$$

Since $H$ is presumed transitive it follows that $\Phi$ is constant. Corollary 3 now applies since the constant, mutatis mutandi, is either no 
larger or no smaller than two.

The mapping implicit in (14) has many pleasant properties when viewed as a mapping from the space of all n-dimensional norms into itself.

To apply Theorem 2 in other situations it is necessary to possess appropriate sets of isometries. We now give one such example. Recall that a norm on $\mathbb{R}^{n}$ is absolute if $\|x\|=\|\mid x\| \|$ for each $x$ in $\mathbb{R}^{n}$. Here $|\cdot|$ is computed component-wise. It is a simple consequence of Caratheodory's theorem that such a norm is actually a lattice norm. Moreover, in this case the mappings $\pi_{k}\left(k=1, \ldots, 2^{n}\right)$,

$$
\pi_{k} y:=\left( \pm y_{1}, \ldots, \pm y_{n}\right)
$$

where the signs range over all permutations of \pm 1 , are linear isometries. This leads to

COROLLARY 8. An absolute norm on $\mathbb{R}^{n}$ is Euclidean if and only if

$$
\frac{1}{2^{n}} \sum_{k=1}^{2^{n}}\left\|x+\pi_{k} y\right\|^{2} \sim 2 \text { for } x, y \in S(X)
$$

Proof. Since $\frac{1}{2^{n}} \sum_{k=1}^{2^{n}} \pi_{k}=0$, the set $P$ of such isometries is balanced with respect to the uniform measure and so Theorem 2 applies.

Obviously the corollary remains true for all balanced subsets of $P$. Finally we observe, that as in [3], certain extensions may be made to replace measures by invariant means.

\section{References}

[1] H. Busemann, The geometry of geodisics (Academic Press, New York, 1955).

[2] M.M. Day, Normed Zinear spaces, Third Edition (Springer-Verlag, Berlin, Heidelberg, New York, 1973).

[3] M.M. Day, "Comments on notes of Stanojević et al", Proc. Amer. Math. Soc. 81 (1981), 554-555. 
[4] A.J. Penico and C.V. Stanojević, "An integral analogue to parallelogram law", Proc. Amer. Math. Soc. 79 (1980), 427-430.

[5] W. Rudin, Functional analysis (McGraw-Hill, New York, 1973).

[6] C.V. Stanojević and A.M. Suchanek, "Integral identities of norms and characterization of inner product spaces", Proc. Amer. Math. Soc. 81 (1981), 101-103.

Department of Mathematics,

Dalhousie University,

Hali fax,

Nova Scotia,

Canada $83 \mathrm{H} 4 \mathrm{H} 8$. 